\title{
IMPACT OF THE DUAL-FREQUENCY SCATTEROMETER ON NOAA OPERATIONS
}

\author{
Zorana Jelenak and Paul S. Chang
}

\section{NOAA/NESDIS/STAR}

\begin{abstract}
In an effort to establish an operational ocean surface vector wind satellite capability, NOAA has been exploring the possibility of flying a U.S. scatterometer on board the Japan Aerospace Exploration Agency's (JAXA's) Global Change Observation Mission (GCOM) satellite series. The Dual Frequency Scatterometer (DFS) has been designed by NASA's Jet Propulsion Laboratory (JPL) and proposed as a baseline scatterometer onboard the GCOM-W2 satellite. This study documents the impact that the DFS instrument will have on different National Weather Service (NWS) weather forecasting and warning products and services. With a $50 \%$ improvement in the accuracy of wind estimates in high wind regimes, a $20 \%$ improvement in resolution and its ability to see through rain, DFS will address NWS's operational OSVW requirements significantly better than a QuikSCAT-like instrument. It is expected that DFS data will have a medium to high impact for all marine weather and tropical cyclone analysis and warning applications, real time diagnostics and climatological wind applications for which wind data are necessary.
\end{abstract}

\section{INTRODUCTION}

NOAA is exploring the possibility of flying a Dual Frequency Scatterometer (DFS) on JAXA's Global Change Observation Mission (GCOM) satellite series. JAXA's GCOM consists of a carbon cycle series (GCOM-C) and water cycle series (GCOM-W) as a successor to their Advanced Earth Observation Satellite (ADEOS) missions. The GCOM-W satellite series is planned to span at least 13 years, with a series of three five-year satellite missions. These satellites will be launched with a one year overlap on successive satellites planned. GCOM-W1 is scheduled for launch in November 2011 followed by GCOM-W2 in January 2016. In addition to a scatterometer, the GCOM-W satellites will carry the next generation AMSR radiometer system.

\section{DUAL FREQUENCY SCATTEROMETER (DFS) DESIGN}

The DFS designed by JPL for the GCOM-W2 satellite is a scanning pencil-beam scatterometer with a $360^{\circ}$ field of view similar to QuikSCAT. The two incidence angles will be slightly different than those of QuikSCAT to preserve the $1800-\mathrm{km}$ wide swath at the $700-\mathrm{km}$ altitude of GCOM-W. Additional DFS details are:

- Ku-band real-aperture radar operating at $13.4-\mathrm{GHz}$ frequency at two polarizations ( $\mathrm{V}$ and $\mathrm{H}$ ) and two incidence angles.

- C-band real aperture type radar operating at $5.4-\mathrm{GHz}$ frequency at H-polarization and two incidence angles.

The characteristics of DFS instrument design and measurement geometry are compared with QuikSCAT and the Extended Ocean Vector Wind Mission (XOVWM) [1] instruments in Fig. 1 and 2.

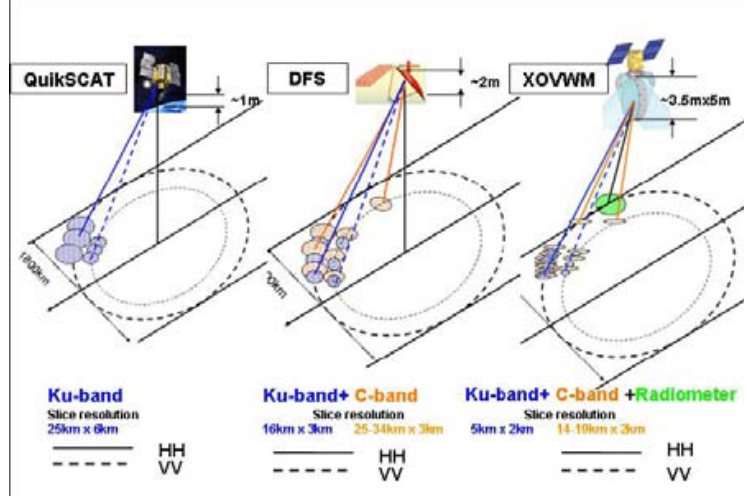

Fig.1 Measurement geometry of QuikSCAT, DFS and eXtended Ocean Vector Wind Mission (XOVWM) instruments.

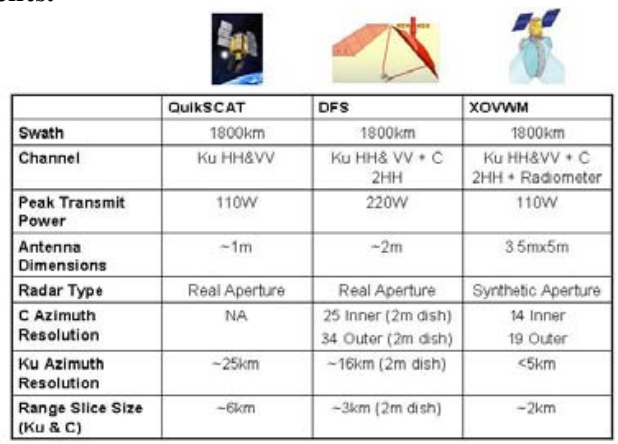

Fig. 2 Characteristics of QuikSCAT, DFS and XOVWM instruments.

\section{OPERATIONAL OSVW REQUIREMENTS AND DFS PERFORMANCE}

\subsection{Spatial Resolution}


The spatial resolution of the radar measurement is determined by antenna size and range resolution. The antenna footprint on the ocean surface is comprised of a series of measurement slices as presented in Fig. 3.

- For a 2-m antenna size, the expected resolution of DFS wind vectors is approximately a $20 \%$ improvement $(\sim 10$ $\mathrm{km}$ ) over QuikSCAT

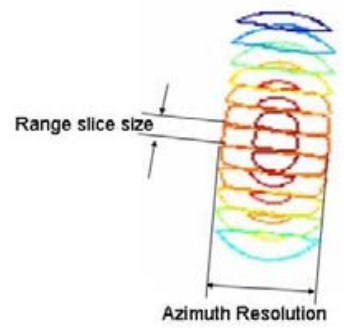

Fig 3. DFS Slice Geometry: Ku - band, 3-km range, 16-km azimuth, C-band, 3-km range, 24-km inner beam, 34-km outer beam

\subsection{All-weather retrievals}

A C-band channel is necessary to improve retrievals performance in rain, and DFS will have an H-pol C-band channel at both incidence angles.

- In the presence of rain QuikSCAT over predicts winds in the low-to-moderate wind speed range. Our experience with ASCAT and the DFS simulations show that the addition of a C-band channel will yield substantial improvements over QuikSCAT retrievals in the presence of rain.

- C-band measurements collected during the NOAA P-3 IWRAP experiments in addition to our experience with ASCAT and the XOVWM and DFS simulations suggest that the C-band measurements are much less affected by rain, and using both $\mathrm{C}$-band and $\mathrm{Ku}$-band channels are necessary to produce wind vector retrievals unaffected by rain

- In clear-sky conditions the DFS retrieval performance will be improved over QuikSCAT by a minimum of $20 \%$ for winds up to $20 \mathrm{~m} / \mathrm{s}$ and by more than $40 \%$ for winds higher than $20 \mathrm{~m} / \mathrm{s}$ (Fig 5)

- The improved performance is due in part to increased power, number of looks, and additional frequency diversity QuikSCAT high wind speed retrievals are limited by both the QuikSCAT measurement frequency (Ku-band) and the QuikSCAT measurement resolution.

- C-band H-pol still has sensitivity as wind speed increases, however the high wind retrievals will still be limited by the spatial resolution of the DFS measurements (Fig. 3 and Fig. 4).

- We expect that the performance of DFS in high-wind regimes will be improved by $50 \%$ over QuikSCAT and that DFS will accurately be able to measure the maximum winds in category 1 and 2 hurricanes (up to about $45 \mathrm{~m} / \mathrm{s}$ ).

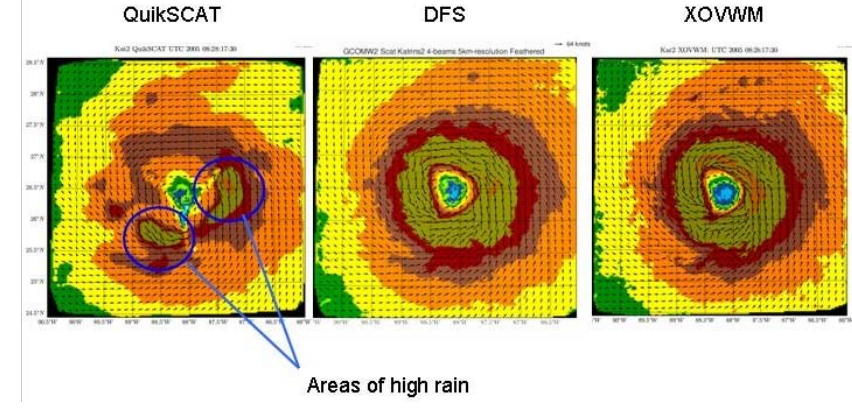

Fig. 4 Simulated QuikSCAT, DFS and XOVWM wind vector retrievals for Katrina-like hurricane.

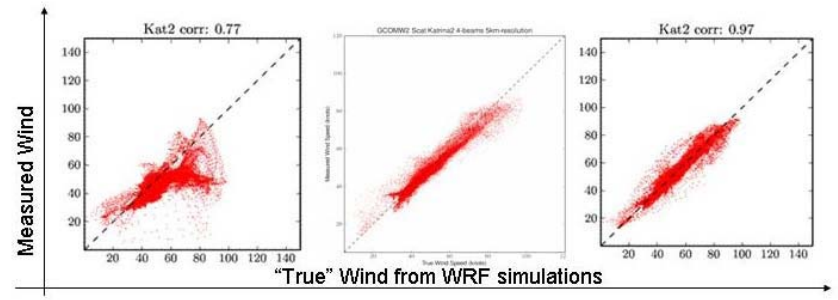

Fig. 5. Scatter plot of retrieved wind speeds in Hurricane Katrina simulations by QuikSCAT, DFS and XOVWM. Underestimation of high wind at $\sim 60 \mathrm{kt}(30 \mathrm{~m} / \mathrm{s})$ is apparent in the case of QuikSCAT. The underestimation of winds with DFS starts to show at $\sim 90 \mathrm{kt}(45 \mathrm{~m} / \mathrm{s})$ due to the spatial resolution of DFS.

\section{IMPACT ON NWS PRODUCTS AND SERVICES}

Table 1. Impact of DFS OSVW on NWS product and services that are using QuikSCAT data today

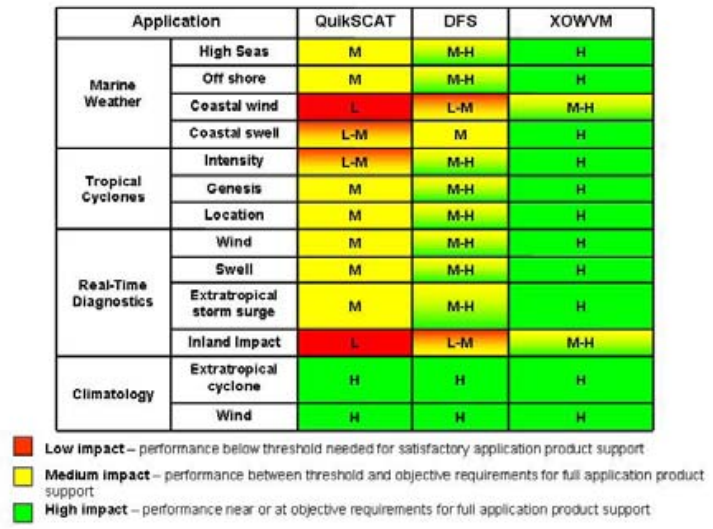

4.1. Marine weather - high seas, offshore, and arctic areas - impact: medium to high

DFS's higher resolution, dual frequency, oversampling, and increased power will yield significantly improved wind retrievals compared to QuikSCAT in all wind conditions, especially in high winds. Wind retrievals from DFS will also show marked improvement over those from QuikSCAT in raining conditions. The improved high-wind capability of 
DFS would give forecasters the ability to see winds well into the hurricane-force range, as high as category 2 intensity (83-95 kt). Unlike with QuikSCAT, where forecasters focused on estimating the appropriate warning category, DFS would allow forecasters to focus on the maximum winds in extratropical cyclones. DFS would mitigate many of the limitations of QuikSCAT in raining conditions, allowing for a more accurate assessment of ocean surface vector winds in all weather conditions. For example, the wind field structure associated with features such as squall lines, fronts, areas of convection, and developing low pressure systems would be much better defined in DFS than with QuikSCAT. Radii of 34-kt, 48-kt, and 64-kt winds speeds in extratropical cyclones would be better defined resulting in improved short-term warnings, especially at higher wind speeds.

For tropical and sub-tropical marine analysis and forecasting applications at NHC and WFO Honolulu, DFS wind retrievals would provide significantly improved ocean vector wind observations of critical features such as tropical waves, fronts, shear lines, subtropical and extratropical cyclones, and the intertropical convergence zone (ITCZ), where wind retrievals from QuikSCAT are often degraded by heavy rain. More accurate wind retrievals in rain and the higher spatial resolution offered by DFS will improve forecaster identification of high-wind regions and areas of wave and swell generation, resulting in improvements across the entire suite of both NHC's and WFO Honolulu marine products. Improved wind retrievals will also increase the accuracy and confidence in surface analysis and in the evaluation of model forecasts for key weather features and their associated wind events. The enhanced spatial resolution of DFS relative to QuikSCAT would also improve the identification, delineation and intensity analysis of areas impacted by and intensity mesoscale wind events occurring within NHC's and WFO Honolulu's areas of responsibility.

The increased resolution of DFS as compared to QuikSCAT is a significant improvement, but DFS wind retrievals would still be limited in some smaller weather features like low pressure systems with strong winds (e.g., high latitude polar lows), convective systems such as MCSs, and small-scale low pressure systems. Ice and ice edge detection would be improved due to the higher resolution and increased power of DFS. Temporal sampling and areal coverage would be the same as for QuikSCAT.

In essence, DFS would give NWS High Seas and Offshore forecasters an improved wind warning capability across a broader range of features than is currently available from the current combination of QuikSCAT and ASCAT.

4.2 Tropical cyclones - intensity, genesis, location impact: medium-high

The proposed Dual Frequency Scatterometer would positively impact analysis, forecast, and warning operations in the tropical cyclone (TC) program at the National Hurricane Center (NHC) and Central Pacific Hurricane Center (CPHC).

Better estimates of TC intensity from DFS would have a significant impact on NHC's and CPHC's tropical cyclone programs. Improvements in the quality of DFS wind retrievals over what is currently available from QuikSCAT, particularly in areas of heavy rain, would provide the ability to accurately estimate the intensity of TCs from tropical depressions to category 2 hurricanes (83-95 kt). This information would be especially beneficial for TCs not sampled by aircraft reconnaissance and when making decisions about upgrading systems to tropical storm or hurricane intensity. More accurate wind direction retrievals from DFS would also improve TC center identification and location fixing, which is critical for determining the initial motion of a cyclone for human forecasters and for the initiation of model guidance. Improved DFS wind directional retrievals would also help determine if a TC has formed or dissipated, and could be used as justification to initiate or discontinue TC advisories, especially when and where aircraft reconnaissance is not available to investigate such systems. This would allow for an earlier issuance of TC advisories and warnings for developing systems. Another major improvement from DFS would be more accurate analysis of the $34-\mathrm{kt}, 50-\mathrm{kt}$, and $64-\mathrm{kt}$ wind radii in all TCs. This information is critical to the placement and timing of coastal watches and warnings and the definition of ship avoidance areas. Currently, QuikSCAT cannot accurately estimate the 64-kt radii in TCs, and estimates of 34-kt and 50-kt wind radii from QuikSCAT are often degraded by rain in all but the strongest hurricanes.

\subsection{Real-time diagnostics - wind, swell, extratropical storm surge, high surf, inland impacts, impact: medium to high, low to medium for inland impacts}

Ocean surface vector winds (OSVW) from QuikSCAT and ASCAT are used by forecasters as a measure of "sea" truth over data-sparse ocean areas of responsibility. This "sea" truth is compared to NWP model short-term forecasts and analyses to assess the validity of NWP guidance for the atmosphere and ocean and determine how much credence (or uncertainty) to ascribe to NWP initializations and forecasts. For example, in a situation where OSVW data show that an extratropical cyclone developing off of the U.S. West Coast is stronger and developing more quickly than NWP analyses and short-term forecasts suggest. Forecasters would then adjust the raw NWP fields based on the OSVW "sea" truth. In this sense OSVW data are used both as a real-time diagnostic tool by which the validity of NWP fields are assessed as a tool by which to adjust forecasts and warnings based on the differences between the "sea" truth and NWP guidance. This application of OSVW data pertains to wind field predictions, the generation of 
wind waves and swell, extratropical storm surge, and even coastal and even inland impacts from high winds and flooding, as well as high surf along the coast. As a result of improved swell identification using DFS, improved forecasts of high surf and potential coastal flooding and beach erosion along coastlines could result in increased lead time and accuracy of coastal WFO's high surf advisories and warnings. An improved OSVW capability from DFS would provide higher quality data which the human forecaster could use to compare NWP fields to OSVW "sea" truth as compared with what can be currently achieved with QuikSCAT and ASCAT.

\subsection{Climatology- extratropical cyclones, wind - impact: high}

The first high-resolution, observationally-based, online interactive atlas of global OSVW has been created from QuikSCAT measurements. This atlas provides highly accurate, global information on wind statistics throughout the Earth's oceans. These data are especially important in regions of the world where there are few ships and buoys to gather data.

Researchers have also compiled nine years of QuikSCAT data to create a never-before-available monthly atlas of how frequently high winds occur over the open ocean all over the world. High winds play an important role in Earth's climate; they remove heat from the ocean, leading to the formation of "deep water" cold, salty, dense water that helps drive global ocean circulation patterns. High winds also help exchange gases, such as carbon dioxide, between the oceans and the atmosphere, mix different types of ocean water, and pump nutrients up from the deep sea for plankton to feed on.

OPC has created a nine-year climatology of hurricane force wind events within North Pacific and Atlantic extratropical storms while TAFB has produced climatology of gale- and storm-force wind events within gap wind events in the Gulf of Tehuantepec. These climate records would not be possible without remotely-sensed OSVW data.

Improved wind retrievals from DFS would also improve the climate data record for the occurrence and intensity of TCs beyond that which is currently available from QuikSCAT. These data would be particularly important in basins where aircraft reconnaissance is not available to make direct measurements of TC intensity.

Winds over the ocean are the largest source of momentum for the ocean surface, and as such they affect the full range of ocean movement - from individual surface waves to complete current systems. OSVW also play key role in regulating the Earth's water and energy cycles by modulating air-sea exchanges of heat, moisture, gases (such as carbon dioxide), and particulates. This modulation regulates the interaction between the atmosphere and the ocean, which establishes and maintains both global and regional climates.

OSVW are required to compute air-sea fluxes and are used in numerical modeling of the ocean and atmosphere for weather and wave forecasts, biophysical interactions and climate studies. As such, characterization and quantification of the role of the global ocean as a planetary heat and carbon sink depends critically on the accurate representation of the global OSVW.

The DFS OSVW measurements will therefore directly supports climate services by providing measurements needed to 1) document heat uptake, transport, and release by the ocean; 2) document ocean carbon sources and sinks (ocean surface vector winds modulates air-sea exchanges of gases such as CO2) and 3) document the air-sea exchange of water and the ocean's overturning circulation.

\section{ECONOMIC IMPACT OF OSVW}

Observations (nowcasts) and forecasts of ocean surface wind conditions are of economic value to industries and activities such as maritime transportation, commercial fishing, offshore energy, recreational boating, and search and rescue. In this paper, we estimate the value of ocean surface wind information generated from satellite-based instruments [2].

Table 2 summarizes our estimates of annual values that can be generated by the use of ocean surface wind observations, nowcasts, and forecasts. We report a range of annual value $\mathrm{s}$ loosely associated with a range of wind information from $t$ hat presently available from QuiSCAT to the more sophistic ated data available with a hypothetical instrument such as $\mathrm{X}$ OVWM.

Table 2. Summery of economic impact of OSVW by Kite-Powell

\begin{tabular}{|c|c|c|c|c|}
\hline \multirow[b]{2}{*}{ Industry/activity } & \multirow[b]{2}{*}{ Source of benefit } & \multicolumn{3}{|c|}{ Annual value, satellite wind obs, $5 \mathrm{~m} /$ year } \\
\hline & & $\begin{array}{l}\text { Low range } \\
\text { (QuikSCAT) }\end{array}$ & $\begin{array}{c}\text { Mid range } \\
\text { (DFS) }\end{array}$ & $\begin{array}{l}\text { High range } \\
\text { (xonwM) }\end{array}$ \\
\hline Maritime transportation & \begin{tabular}{|l} 
Reduced exposure to \\
eatrotropical storms \\
\end{tabular} & 196 & 187 & 202 \\
\hline USCG Search \& Rescue & $\begin{array}{l}\text { Improved search } \\
\text { etficiency, lives soved }\end{array}$ & 1 & 4 & 29 \\
\hline Otfshore energy & $\begin{array}{l}\text { Recused downtime, } \\
\text { driling and production }\end{array}$ & 7 & 9 & 12 \\
\hline Commercisl fishing & $\begin{array}{l}\text { Reduced exposure to } \\
\text { hszardous conditions }\end{array}$ & $<1$ & 1 & 11 \\
\hline Recrestionsl Dosting & $\begin{array}{l}\text { Reduced exposure snd } \\
\text { improved pssssge } \\
\text { plsnning }\end{array}$ & $<1$ & 1 & 3 \\
\hline
\end{tabular}

\section{REFERENCES}

[1] Gaston, R. and E. Rodriguez, "QuikSCAT Follow-On Concept Study”, JPL Publication, no. 18-8, pp. 60, April 2008

[2] H.L. Kite-Powell, 2009. The role of ocean surface wind information in mitigating risk to maritime transportation. Maritime Economics and Logistics. In review 